\title{
Caninos impactados: revisão de literatura
}

\section{Impacted canines: literature review}

Recebido: 10/11/2021 | Revisado: 12/11/2021 | Aceito: 30/12/2021 | Publicado: 01/01/2022

Caleb Benjamin Soto Arispe

Universidade Brasil, Brasil

E-mail: calebsoto03@gmail.com

Emely Janaine Layme Marca

Universidade Brasil, Brasil

E-mail: emelyjanaine1801@gmail.com

José Lucas Martins

Universidade Brasil, Brasil

E-mail: lucas.martins@universidadebrasil.edu.br

\begin{abstract}
Resumo
O tratamento de caninos impactados é um grande desafio para os cirurgiões dentistas. Os caninos permanentes exercem um papel importante da dentição, funções na conformação da arcada dentária humana, tanto do ponto de vista estético como funcional. Para a sua erupção do canino superior percorre um caminho longo e complexo, deslocando-se desde a parte lateral da fossa piriforme, local de formação, até a sua posição na arcada. O tempo de percurso até o local de erupção é duas vezes superior aos restantes dentes e sua formação tardia torna-se mais susceptível a sofrer alterações na trajetória de erupção tornando-se impactado. Portanto torna-se importante estudar todas as abordagens que solucionem essa patologia. Sua etiologia está relacionada à falta de espaço no arco dentário. Seu tratamento requer uma abordagem cautelosa da interpretação de um Ortodontista e Cirurgião Buco Maxilo Facial. Exames de imagens são primordiais e o prognóstico depende da posição do canino em relação às estruturas adjacentes.
\end{abstract}

Palavras-chave: Caninos permanentes; Dente canino; Dente impactado.

\begin{abstract}
The treatment of impacted canines is a great challenge for dental surgeons. Permanent canines play an important role in dentition, functions in the conformation of the human dental arch, both from an aesthetic and functional point of view piriform, formation site, until its position in the arch. The travel time to the eruption site is twice as long as the remaining teeth and its late formation becomes more susceptible to change in the eruption site is twice as long as the remaining teeth and its late formation becomes more susceptible to change in the eruption trajectory, becoming impacted. Therefore, it is important to study all approaches that solve this pathology. Its etiology is related to the lack of space in the dental arch. Its treatment requires a cautious approach to interpretation by an Orthodontist and Oral and Maxillofacial Surgeon. Imaging exams are essential and the prognosis depends on the position of the canine in relation to adjacent structures.
\end{abstract}

Keyword: Canines permanent; Canine tooth; Impacted tooth.

\section{Introdução}

Os caninos superiores permanentes são dentes que desempenham importantes funções na conformação da arcada dentária humana, tanto do ponto de vista estético como funcional. Seu papel é fundamental na oclusão, suportando os movimentos de lateralidade, a carga de mastigação.(Campos et al, 2020)

A impactação de caninos superiores é um acontecimento frequente, para sua erupção é necessário percorrer um caminho longo e complexo, deslocando-se desde a parte lateral da fossa piriforme, local de formação até a sua posição na arcada. (Almeida et al, 2001)

O tempo de percurso até o local de erupção é duas vezes superior aos restantes dentes, é um dos últimos dentes a irromper na cavidade bucal, sendo tardia pode ocasionar a falta de espaço no arco dentário, susceptível a sofrer alterações na trajetória de erupção, podendo levar à sua inclusão. Pode sofrer alteração de erupção desde a odontogênese até o estabelecimento da oclusão normal. Isto pode acabar resultando em uma erupção ou impactação por vestibular ou palatino.(Almeida et al, 2001) 
Por motivos multifatoriais, quando chegada a época de erupção o dente pode ficar recluso parcial ou totalmente no interior do osso, com manutenção ou não da integridade do saco pericoronário, se caracteriza como um dente retido/incluso ou impactado. (Marzola et al, 1995.)

Dentre os dentes mais acometidos por essa anomalia, encontram-se os terceiros molares e os caninos permanentes (Mittal et al., 2013)

As impactações palatinas são mais prevalentes (85\%) do que os vestibulares (15\%) (Fonseca et al., 2016). Em alguns estudos, observou-se que os caninos superiores e inferiores inclusos ocorrem em $2 \%$ e $0,2 \%$ da população, respectivamente, sendo a frequência de inclusão entre caninos permanentes 10 vezes maior na maxila do que na mandíbula (Da Silva et al., 2018).

A literatura aponta como principais causas de retenção de caninos os fatores hereditários, a raça, os distúrbios endócrinos e as síndromes com mal formação craniofaciais. (Alves, et al 2014)

A impactação de caninos superiores é um acontecimento frequente, especialmente na região palatina, ainda que na presença de espaço insuficiente para o seu alinhamento na arcada dentária. Pode ser causada por fatores de ordem geral ou local, sendo que seu diagnóstico deve ser realizado por meio de exames clínicos e radiográficos específicos.Também se deve levar em consideração a possibilidade do canino impactado não se movimentar ortodonticamente. Neste caso, será necessária a sua extração e o espaço poderá ser ocupado pelo pré molar ou por uma prótese. (Cappellette 2008)

A frequência em mulheres é três vezes maior do que em homens.Complicações que podem ser causadas por caninos impactados: mau posicionamento labial ou lingual ,migração dos dentes vizinhos e perda do comprimento do arco, reabsorção interna, formação de cisto dentígero, reabsorção externa da raiz do dente afetado dente impactado, e dentes vizinhos, infecção particularmente com erupção parcial, e sensação de dor (Neville, 2009)

\section{Metodologia}

Foi realizada uma revisão de literatura, com busca nas bases de dados: Scielo, PUBMED, Google Scholar. Foram pesquisadas palavras como "Caninos Impactados ,Caninos no palato" Foram selecionados 20 artigos nos idiomas: inglês, espanhol e português com recorte temporal do 1936 de 2021.

\section{Resultados Discussão}

\section{Etiologia}

Para Kaczor et al. (2016) a etiologia da impactação dentária é multifatorial apresenta etiologias distintas dependendo da face em que a impacção ocorre, na literatura, os fatores etiologias relacionadas à impactação são divididas em três grupos diferente: sistêmica, local e genética. Fatores sistêmicos: deficiências endócrinas (por exemplo, hipotireoidismo), radioterapia, disostose cleidocraniana, amelogênese imperfeita. Fatores locais: falha na reabsorção do dente decíduo, perda prematura de um dente decíduo, retenção prolongada de um dente decíduo, caminho eruptivo anormal, presença de dentes supranumerários, aglomeração dentária e perda de espaço, extração precoce de dente decíduo, folículo dentário aumentado/cisto dentígero ou outras formas de patologia dos tecidos moles (neoplasia), reparação espessada pós-extração ou pós trauma da mucosa, traumatismo dentário, odontoma, anomalia na posição de um dente (por exemplo, inclinação, deslocamento, transmigração), anquilose de molares decíduos, dilacerações radiculares, fenda alveolar. Fatores genéticos: fatores hereditários, como um germe dentário mal posicionado e presença de uma fenda alveolar.

Almeida et al.(2001), as causas podem ser primárias e secundárias. Enumerou como causas primárias:1) reabsorção radicular do dente decíduo; 2) trauma dos germes dos dentes decíduos; 3) disponibilidade de espaço no arco; 4) rotação dos germes dos dentes permanentes; 5) fechamento prematuro dos ápices radiculares; 6) irrupção de caninos em áreas de fissuras 
palatinas; e como causas secundárias: 1) pressão muscular anormal; 2) doenças febris; 3) distúrbios endócrinos; e 4) deficiência de vitamina D.

Há autores que defendem que o posicionamento ectópico do canino esteja fortemente ligado a fatores genéticos. Peck et al.(1994) , afirmaram que a etiologia do deslocamento da maioria dos caninos para palatino e sua subseqüente impactação é primariamente de origem genética. Segundo os autores, este fenômeno costuma ocorrer em associação com outros fatores controlados geneticamente. Dentre eles estão:1) A ocorrência concomitante com outras anomalias dentárias como alterações de forma, tamanho e número de dentes. 2) Relatos de incidência bilateral de 17 a $45 \%$ dos casos de desvio palatino do canino, apontam para o mecanismo genético como fator etiológico intrínseco.3) A ocorrência ligada ao sexo, com prevalência maior para o sexo feminino e em grau semelhante a outras anomalias dentárias de origem genética.4) A incidência alta nos membros de uma mesma família juntamente com outras anomalias dentárias.5) Diferenças de ocorrência em grupos populacionais, com maior incidência em europeus.

\section{Classificação de dentes caninos impactados}

Vertical - os caninos se encontram paralelos em relação ao longo eixo do incisivo lateral; mesioangular - o longo eixo do canino está em posição medial em relação ao longo eixo do incisivo lateral; distoangular - o longo eixo do canino está em posição distal em relação ao longo eixo do incisivo lateral; horizontal - o longo eixo do canino está perpendicular ao longo eixo do incisivo lateral. Alves et al. (2014). A seguir, figura 1 apresenta desenho esquemático do posicionamento dos caninos superiores inclusos adaptado da classificação de Winter para terceiros molares.

Figura 1 - Classificação.

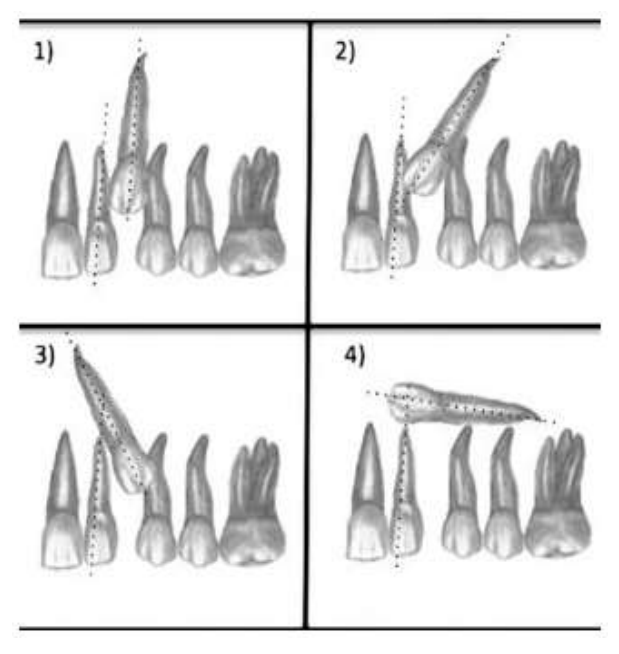

Fonte: Scielo. Alves, Eduardo Peterini (2014).

\section{Diagnóstico}

Para a realização de um diagnóstico preciso, torna-se necessária a associação dos exames clínicos (inspeção e palpação) com os radiográficos.

No exame clínico, alguns sinais podem indicar a presença de caninos impactados: atraso na irrupção de um ou mais caninos, após os 14 anos de idade; retenção prolongada de caninos decíduos; abaulamento do tecido mole por palatina ou vestibular; migração distal dos incisivos laterais com ou sem desvio da linha média. A palpação também é usada geralmente, em $70 \%$ dos casos, um dente impactado pode ser palpado. Outro meio de Diagnóstico são os exames radiográficos com diferentes técnicas radiográficas, com o intuito de localizar os caninos não irrompidos. As mais comuns são: radiografias periapicais, 
radiografias oclusais, radiografias panorâmicas, telerradiografias em norma lateral e frontal. (Almeida 2001)

\section{Localização tomografica}

Como prevenção a impactação, uma investigação radiográfica é ideal como auxiliar de diagnóstico entre 9 e 13 anos de idade, por meio de uma panorâmica podemos observar os corredores de erupção e a rizólise do canino decíduo. (Silva 2009)

Primeiro sugerir a extração interceptava do canino decíduo como forma de facilitar a erupção de caninos superiores palatinamente desviados e evitar o risco de reabsorção radicular. (Buchner 1936).

Aos 8 anos de idade, na falta da protuberância na região do canino, o diagnóstico com radiografias pode mostrar a posição lingual do canino superior não erupcionado e o risco de impactação, recomendando a remoção precoce do canino decíduo e manutenção do espaço. (Williams 1981)

A extração dos caninos decíduos na dentadura mista, antes dos 11 anos de idade, influencia positivamente a erupção dos caninos permanentes, tendendo a normalizar sua posição em $91 \%$ dos casos, se a coroa do canino estiver posicionada a distal da raiz do incisivo lateral e, em $64 \%$ se a coroa do canino estiver a mesial da raiz do incisivo lateral. (Ericson \& Kurol 1988)

Linhas de referências e medidas lineares e angulares podem ser realizadas na radiografia panorâmica, com objetivo de avaliar a maior ou menor probabilidade de erupção espontânea. (Ericson \& Kurol 1988)

\section{Considerações Finais}

Devido a importância estética e funcional dos caninos no desenvolvimento da oclusão normal de um indivíduo o tratamento de caninos impactados no palato é necessário para tentar de todas as formas reposicionar estes dentes no arco dentário do paciente se possível.

\section{Agradecimentos}

Agradecemos a Deus, a senhora Elsa Layme, e aos membros da família Soto Arispe e aos professores da Universidade Brasil em especial ao professor José Lucas Martins por nos ter auxiliado na realização deste artigo.

\section{Referências}

Almeida, G. M., Cavalcanti, R. B. de M. S., Medeiros, G. de S., Almeida, M. D. de A., Castro, R. M., Andrade, M. A., Gomes, L. L., Moura, A. B. R., Maia, L. S., Gomes, E. N. S., Arruda, L. F. de A., Freitas, G. B. de, Ribeiro, E. D., \& Rocha, J. F. (2020). Use of included teeth: case reports. Research, Society and Development, 9(7), e653974585. https://doi.org/10.33448/rsd-v9i7.4585.

Almeida, R. R., Fuziy, A., Almeida, M. R., Almeida Pedrin, R. R., Henriques, J. F. C., Insabralde, C. M. B. (2001). Abordagem da impactação e/ou irrupção ectópica dos caninos permanentes: considerações gerais, diagnóstico e terapêutica. Rev. Dental Press Ortodon. Ortop. Facial, Maringá, 6(1), p. 93-116.

Alves, E. P.; Montagner, A. F.; Antoniazzi, S. P. \& Oliveira, L. F. D. (2014). Prevalência e posição de caninos superiores impactados e sua relação com reabsorção radicular. RFO UPF 19(2), 180-184.

Bassi, A. P. F., Tonini, K. R., \& Ponzoni, D. (2021). Primary retention of permanent lower first molar: case report and twelve-year follow-up. Research, Society and Development, 10(4), e24110413699.https://doi.org/10.33448/rsd-v10i4.13699.

Buchner, H. J. (1936). Reabsorção radicular causada pela erupção ectópica da cúspide maxilar. Jornal Internacional de Ortodontia e Cirurgia Oral, 22, 12361238 .

Campos, M. S., Nascimento, M. C., Machado, M., Vargas, L. R., Pimentel, R.M. (2020). Exodontia de canino incluso por meio de retalho palatino - relato de caso, Ciência Atual, 15(1).

Capellete, M., Capellete Jr. M., Fernades, L. C. M., Oliveira, A. P., Yamamoto, L. H., Shido, F. T., Oliveira, W. C. (2008). R Dental Press Ortodon Ortop Facial, Caninos permanentes retidos por palatino: diagnóstico e terapêutica - uma sugestão técnica de tratamento, 64 Maringá, 13(1), 60-73.

Da Silva, F. J. (2018). Intervenção cirúrgica de um canino incluso em sínfise mandibular: relato de caso. Revista Da Faculdade De Odontologia - UPF, 23(3), 329-332. https://doi.org/10.5335/rfo.v23i3.8613 
Ericson, S., \& Kurol, J. (1988). Resorption of maxillary lateral incisors caused by ectopic eruption of the canines. A clinical and radiographic analysis of predisposing factors. American journal of orthodontics and dentofacial orthopedics : official publication of the American Association of Orthodontists, its constituent societies, and the American Board of Orthodontics, 94(6), 503-513. https://doi.org/10.1016/0889-5406(88)90008-x.

Ericson, S.,, \& Kurol, J.(1988). Tratamento precoce de caninos superiores em erupção palatina por extração dos caninos primários, European Journal of Orthodontics , 10(4), 283-295. https://doi.org/10.1093/ejo /10.4.283.

Fonseca, C., et al. (2016). Métodos de diagnóstico imagenológico para optimizar el plan de tratamiento y pronóstico de caninos maxilares: Imaging diagnosis methods to optimize treatment planning and prognosis of maxillary canines. Revista Estomatologia Herediana, 26(4), p. 263-270, oct. 2016.

Gomes, L. R., Koga, R. S.; Santos, C. P., Gomes, R. L. O., \& Filho M. J. S. F. (2021). Alternativas clínicas no tratamento de caninos impactados: revisão de literatura , Brazilian Journal of Development, Curitiba, 7(2), 11897-11911.

Kaczor, K. U., et al. (2016). Impacted Teeth: An Interdisciplinary Perspective. Advances in Clinical and Experimental Medicine, 25(3), 575-585.

Marzola, C.(1995). Retenção dental. (2a ed.), Ed. Pancast, 13-135.

Mittal, R., Rai, D,. Patil, A., \& Garg, A. (2013). An easy method of attachment to an impacted canine. Progress Orthod, 3, 11 a 14. https://doi.org/10.1186/21961042-14-11

Neville, B. Patologia oral e maxilofacial.(2009). (3a ed.), Editora Elsevier.

Peck, S., Peck, L., \& Kataja, M. (1994). The palatally displaced canine as a dental anomaly of genetic origin. The Angle orthodontist, 64(4), 249-256. https://doi.org/10.1043/0003-3219(1994)064<0249:WNID>2.0.CO;2

Rodrigues, J. P. P., Carvalho, L. R. R. de A., Cravinhos, J. C. de P., Carvalho, L. R. D. A., Moura, R. A., Melo Neto, M. V. de, Teixeira, P. R. S., \& Silva, F. A. D. J. C. (2020). Surgical treatment of impacted canine in the mandible basis: a case report. Research, Society and Development, 9(8), e323985176. https://doi.org/10.33448/rsd-v9i8.5176

Silva Filho, O. G. (2009). Influência da expansão rápida da maxila na rizólise dos caninos decíduos usados como ancoragem. Rev Dental Press Ortodon Ortop Facial,14(5), 53-61.

Williams, B. H.(1981). Diagnosis and Prevention of Maxillary Cuspid Impaction. Angle Orthod 1 January 1981, 51(1), 30-40. https://doi.org/10.1043/00033219(1981)051<0030:DAPOMC >2.0.CO;2 